\title{
The Role of Eye Color in the Emergence of Tinnitus in Silence
}

\author{
Onyinyechi C. Ukaegbe ${ }^{1,2}$ Denise A. Tucker ${ }^{1}$ (1) \\ ${ }^{1}$ Communication Sciences and Disorders Department, School of \\ Health and Human Sciences, University of North Carolina, \\ Greensboro \\ 2 Otorhinolaryngology Department, Faculty of Medical Sciences, \\ University of Nigeria, Enugu
}

\begin{abstract}
Address for correspondence Onyinyechi C. Ukaegbe, MBBS, Ph.D., FWACS, Department of Communication Sciences \& Disorders University of North Carolina Greensboro, 327A Ferguson Building, Greensboro, NC 27402-6170, United States (e-mail: ocukaegb@uncg.edu).
\end{abstract}

\begin{abstract}
Introduction Previous research suggests that African Americans are less likely than Caucasians to perceive tinnitus in sustained silence.

Objective To evaluate the association between non-cutaneous melanin as indicated by eye color and the emergence of temporary tinnitus during a brief period of silence. Methods A cross-section of adults grouped according to their eye color were exposed to silence. A total of 62 adults, aged 18 to 35 years ( 10 males, 52 females) were required to sit in silence for 10 minutes, after which they filled out a questionnaire to report their eye color and any perception of sounds in the ears or head.

Results In total, $63 \%$ of the participants perceived tinnitus while sitting in silence, and, of these $95 \%$ perceived the tinnitus sounds within 5 minutes of sitting in silence. Though African Americans were less likely to perceive tinnitus in silence, this difference was not significant $(p=0.6)$. After a period of silence, $69 \%$ of the subjects with lightcolored eyes and $58 \%$ of the dark-eyed subjects perceived tinnitus. This difference was not statistically significant $\left(x^{2}(1)=0.77 ; p=0.38\right)$.

Conclusion When exposed to reduced auditory stimulation, 3 out of 5 normal-hearing

Keywords

- tinnitus

- eye color

- melanin people are likely to experience tinnitus. However, there was no relationship between eye color and the perception of tinnitus in silence. Although melanin has been shown to play a role in the protection of the ear against noise trauma and the effects of age-related hearing loss, its role in the emergence of tinnitus needs further investigation.
\end{abstract}

\section{Introduction}

Tinnitus is the perception of sound in the absence of an auditory stimulus. ${ }^{1,2}$ It is often reported as the perception of sound in the ears or the head, and this can be unilateral or bilateral. ${ }^{3}$ Tinnitus can be perceived as noise or tones such as ringing, buzzing, humming, whistling, hissing, roaring, cricket-like or like water falling from a height. ${ }^{4-6}$ These can be persistent or intermittent. It is estimated that 50 million Americans experience tinnitus; however, it is persistent in 16 million of these. ${ }^{7,8}$ About $7.2 \%$ of tinnitus sufferers refer to their tinnitus as a big problem, and an estimated $2 \%$ find it debilitating and detrimental to their quality of life. ${ }^{1,9}$ Tinnitus has been associated with anxiety, depression, insomnia, and difficulty concentrating., ${ }^{40,11}$

Tinnitus is suspected to be the result of compensatory changes in the auditory pathway when exposed to perturbations and reduced auditory input. ${ }^{12-15}$ However, there is still a lot that we don't know about the factors that influence received

June 25, 2020

accepted after revision

November 24, 2020

published online

November 11, 2021
DOI https://doi.org/ 10.1055/s-0041-1726039. ISSN 1809-9777.

\footnotetext{
(c) 2021. Fundação Otorrinolaringologia. All rights reserved. This is an open access article published by Thieme under the terms of the Creative Commons Attribution-NonDerivative-NonCommercial-License, permitting copying and reproduction so long as the original work is given appropriate credit. Contents may not be used for commercial purposes, or adapted, remixed, transformed or built upon. (https://creativecommons.org/ licenses/by-nc-nd/4.0/) Thieme Revinter Publicações Ltda., Rua do Matoso 170, Rio de Janeiro, RJ, CEP 20270-135, Brazil
} 
the emergence of tinnitus, such as why it is present in some people with normal hearing, or why it is not universally present in everyone when they are exposed to reduced auditory input. ${ }^{1,16}$ The pathophysiology of tinnitus generation is still unclear, and the treatment of chronic tinnitus remains a challenge.

Silence tends to increase tinnitus perception and awareness in people with tinnitus. To avoid this, people with tinnitus are advised to avoid silence. However, it appears that a majority of adults with no prior history of tinnitus or ear pathology report the perception of temporary tinnitus when exposed to sustained silence. ${ }^{17-19}$ There are several possible explanations for this phenomenon. First, the removal of environmental sounds may result in the perception of sounds that were previously masked by an enriched acoustic environment. Another explanation is that following a period of reduced auditory stimulation, the auditory system temporarily undergoes functional changes that manifest as tinnitus. ${ }^{12}$ If the latter is the case, studying the perception of tinnitus in silence could improve our understanding of why it is present in some people and absent in others with similar thresholds. The differences that exist between those that perceive tinnitus in silence and those that don't are likely to improve our understanding of the epidemiology of tinnitus and the modifying factors in tinnitus emergence. This is especially relevant for tinnitus resulting from reduced auditory input, often seen in hearing loss from cochlea, middle ear and outer ear pathologies.

One possible modifying factor in the perception of tinnitus resulting from reduced auditory stimulation could be melanin. The emergence of tinnitus during sustained silence appears to be less likely in African Americans than in Caucasians. ${ }^{19}$ It has also been reported that non-Hispanic whites are more likely to have frequent tinnitus than other racial groups. ${ }^{8}$ Temporal bone studies have shown that African Americans have higher levels of cochlear melanin than Caucasians, and research has shown that people with darker iris pigmentation have higher levels of melanin in the cochlea. ${ }^{20-24}$ Therefore, higher levels of melanin may offer some protection against the emergence of tinnitus. Melanin is present in the eyes and inner ears, as well as in the skin, in varying proportions that determine the skin color and eye color. ${ }^{25,26}$ Melanin is also present in the brain in the form of neuromelanin. ${ }^{26,27}$ The same genes that determine eye color are involved in the regulation of melanin, and the level of melanin in the ear can be estimated from eye color. ${ }^{23,24}$ The melanin-containing cells synthesized in the intermediate layer of the stria vascularis are thought to actively protect against marginal cell injury and loss. ${ }^{28}$ Melanin and its precursors are also thought to play a role in the protection of the inner ear from oxidative stress. ${ }^{29,30}$ Furthermore, melanin is an important reservoir for ion pumps, and plays a role in the maintenance of signal transduction; this is true for inner-ear melanin and neuromelanin, which, although not widely researched on, has been postulated to play a role in normal action potentials in neurons. ${ }^{26,31,32}$ Susceptibility to noise-induced hearing loss and age-related hearing loss appears to be higher in those with lower levels of melanin or lighter eye colors; furthermore, research has shown that melanin plays a role in ototoxicity, and that certain drugs reduce the perception of tinnitus by binding to melanin within the cochlea. ${ }^{14,15,28,30,33,34}$ Collectively, this suggests that people with higher levels of extracutaneous melanin are less susceptible to the effects of auditory perturbations or injury, and, therefore, less likely to perceive tinnitus. Since we cannot always explain why some people have tinnitus, the study of the role of extracutaneous melanin in tinnitus perception could help improve the current knowledge of the possible risk factors for tinnitus emergence and enrich the existing knowledge of the epidemiology of tinnitus. Additionally, understanding the role of melanin in tinnitus generation will be useful in estimating the individual efficacy of any future tinnitus therapy that targets melanin's ability to bind to chemicals.

The purpose of the present study was to follow up previous research which showed that African Americans were less likely to perceive tinnitus in silence, and to further explore the role of melanin in the emergence of tinnitus after a brief period of sustained silence. This is a role that has previously been explored with respect to noise-induced hearing loss and age-related hearing loss. In the present study, the sounds perceived in silence are referred to as tinnitus, and eye color was used to estimate the level of inner-ear melanin; it is hypothesized that those with lighter eye colors will be more likely to perceive tinnitus in silence than those with darker eye colors. The present study hopes to throw more light on the influence of melanin on tinnitus emergence following reduced auditory stimulation.

\section{Methods}

The participants were 62 adults, 10 males and 52 females, aged 18 to 35 years, who had given their consent to participate in the study. Participants were only included in the study if they had no history of chronic tinnitus, head trauma, neurological disease, ear surgery, or recent ear infections. We studied adults with normal hearing thresholds instead of tinnitus patients in an effort to simulate the effect of sensory deprivation on an intact auditory pathway. This reduces the heterogeneity that often affects studies with tinnitus subjects who frequently have varying sites of auditory pathology. ${ }^{35}$ The present was a descriptive cross-sectional study, and approval for it was obtained from the Institutional review board under IRB Number is 18-0066. Fliers and emails were used in the recruitment of participants and convenience sampling was employed. The study was conducted over a two-year period. The sample size was calculated using the effect size and power from similar studies. ${ }^{17,36}$ The participants were initially told that the study was about their experience in silence; their attention was not drawn to their auditory system, and tinnitus was not mentioned.

The participants were asked to self-identify their eye color, and 10 (16\%) reported that their eyes were either blue or gray, 16 (26\%) had green or hazel eyes, and 36 (58\%) had brown eyes. All participants had pure-tone hearing thresholds of $25 \mathrm{dBHL}$ or less at octave frequencies between $250 \mathrm{~Hz}$ and $8,000 \mathrm{~Hz}$, with type-A tympanograms and 
Table 1 Subject demographics

\begin{tabular}{|c|c|c|c|}
\hline & & Frequency & Percentage \\
\hline \multirow[t]{7}{*}{ Race } & Non-Hispanic White & 32 & 51.6 \\
\hline & African Americans & 21 & 33.9 \\
\hline & Hispanic & 4 & 6.5 \\
\hline & Middle-Eastern & 2 & 3.2 \\
\hline & Native American & 1 & 1.6 \\
\hline & Other & 2 & 3.2 \\
\hline & Total & 62 & 100.0 \\
\hline \multirow[t]{3}{*}{$\begin{array}{l}\text { Eye } \\
\text { Color }\end{array}$} & Light eye color & $\begin{array}{l}26 \text { (5 males, } \\
21 \text { females) }\end{array}$ & 41.9 \\
\hline & Dark eye color & $\begin{array}{l}36 \text { (5 males, } \\
31 \text { females) }\end{array}$ & 58.1 \\
\hline & Total & 62 & 100.0 \\
\hline \multirow[t]{3}{*}{ Gender } & Male & 10 & 16.1 \\
\hline & Female & 52 & 83.9 \\
\hline & Total & 62 & 100.0 \\
\hline
\end{tabular}

normal findings on otoscopy. Those who met the inclusion criteria were asked to sit quietly inside a sound-proof booth for a period of ten minutes. We made use of ten minutes of sustained silence because previous research had shown that most participants report an early emergence of tinnitus within the first five minutes, when left in silence. ${ }^{19}$

The noise levels within the sound-proof booth were within American National Standards Institute's recommendations. Subsequently, the participants completed a short Qualtrics Survey to answer questions about the emergence of tinnitus perception and give a description of the type of sound heard, where it was perceived (ears and/or head), and when it was perceived. The participants that perceived tinnitus while in silence also provided information about the number of sounds heard and the pitch of the tinnitus. Tinnitus perception was defined as sounds heard in the ears and/or the head while sitting in silence.
The participants were divided into two groups according to eye color. The light-eyed group consisted of those with blue, gray, green and hazel eyes, while the dark-eyed group consisted of participants with brown and black eyes. The International Business Machines (IBM) Statistical Package for the Social Sciences (SPSS) Statistics (IBM Corp., Armonk, NY, US), version 25, was used in the data analysis. The Chisquared $\left(X^{2}\right)$ test or its non-parametric equivalent was used in testing for any association between eye color and race, and tinnitus perception $t$-tests were used to examine if the onset of tinnitus perception differed by eye color.

\section{Results}

\section{Demographics}

- Table 1 shows the distribution of the participants by race, eye color and gender. The mean age of the participants was $22.92 \pm 3.5$ years.

\section{Perception of Tinnitus during a Brief Period of Silence}

In total, $63 \%$ of the participants perceived tinnitus while sitting in silence, and, of these, 95\% perceived the tinnitus sounds within 5 minutes of sitting in silence (-Fig. 1). A total of $54 \%$ of the subjects who perceived tinnitus did so in both ears, and $21 \%$ perceived the tinnitus sound in the head; $45 \%$ of those who perceived tinnitus described their tinnitus as having 1 sound, while $32 \%$ heard 3 or more sounds.

Overall, 28 (74\%) of those who perceived tinnitus while sitting in silence described it as having a pitch, and more than half of these had high-pitched tinnitus (54\%). Only 3 (11\%) thought that their tinnitus was low-pitched.

-Fig. 1 shows the reported onset of tinnitus perception for each eye-color group. A greater proportion of dark-eyed participants perceived tinnitus after one minute of exposure to silence. However, there was no statistically significant difference in the onset of tinnitus perception when both groups were compared $\left(t_{37}=0.72 ; p=0.48\right)$. Both groups were more likely to report that they heard 2 or more sounds while in silence $\left(t_{36}=-0.23 ; p=0.82\right)$.

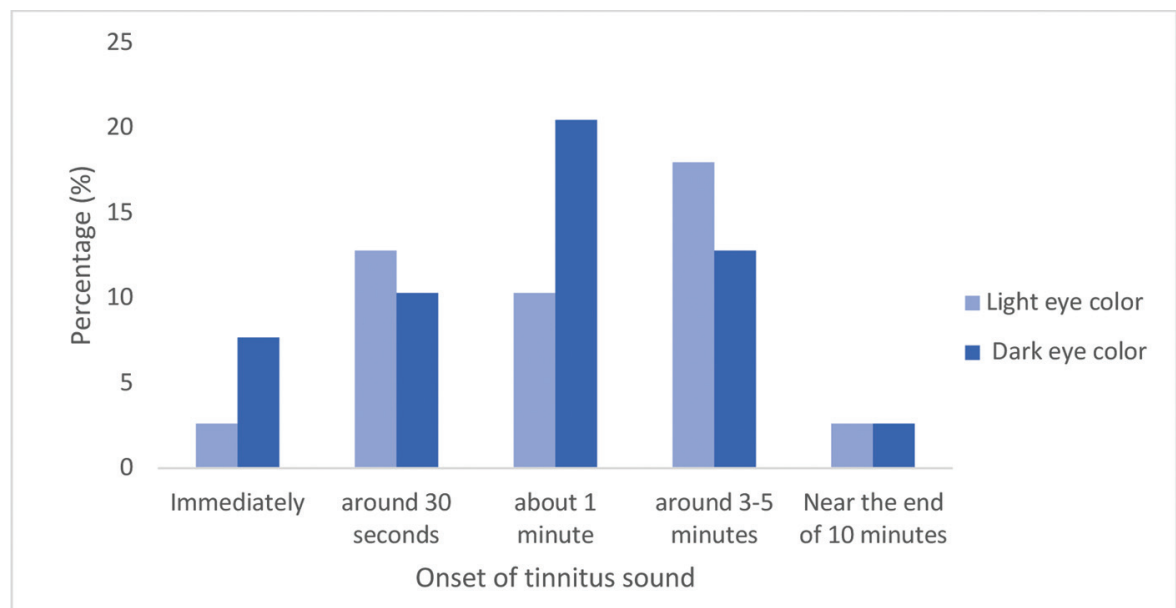

Fig. 1 The onset of tinnitus perception in silence by eye color. 


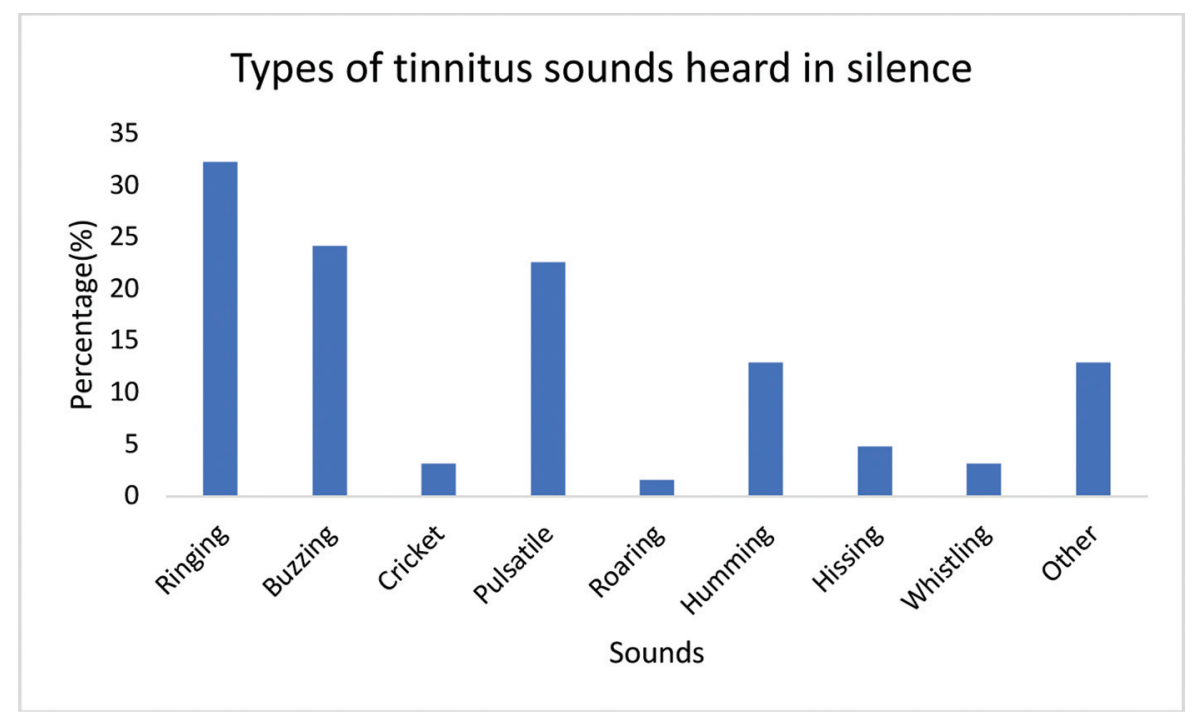

Fig. 2 Types of tinnitus sounds heard.

-Fig. 2 shows the types of sounds heard and the proportion of participants.

\section{Race Distribution of Tinnitus Perception during a Brief Period of Silence}

In total, 23 (72\%) of non-Hispanic whites perceived tinnitus in silence, while 11 (52\%) of African Americans perceived tinnitus in silence. A Fisher exact test did not show a significant association between tinnitus perception and race (Fisher $=3.99 ; p=0.6$ ).

The relative risk of perceiving tinnitus was 1.4 times higher in Caucasians than African Americans. The odds of perceiving tinnitus in silence was two times higher in Caucasians than in African Americans.

\section{Eye Color and Tinnitus Perception in Silence}

Overall, 18 (69\%) of the light-eyed subjects and 21 (58\%) of the dark-eyed subjects perceived tinnitus after a period of silence (-Fig. 3). This difference was not statistically significant $\left(\mathrm{X}^{2}(1)=0.77 ; p=0.38\right)$

The relative risk of perceiving temporary tinnitus in silence was 1.2 times higher in those in the light-eye-color group than in those in the dark-eye-color group. The light-eyed subjects were 2.25 times more likely to perceive tinnitus in silence than not, while the dark-eyed subjects were 1.4 times likely to perceive tinnitus in silence than not. Therefore, the odds of perceiving tinnitus when exposed to silence was 1.6 times higher in the light-eyed group when compared with the dark-eyed group.

\section{Discussion}

\section{Perception of Tinnitus in Silence}

The proportion of participants with normal hearing thresholds who perceived tinnitus in the present study was similar to the $64 \%$ reported by Tucker et al., ${ }^{19} 2005$, but much lower

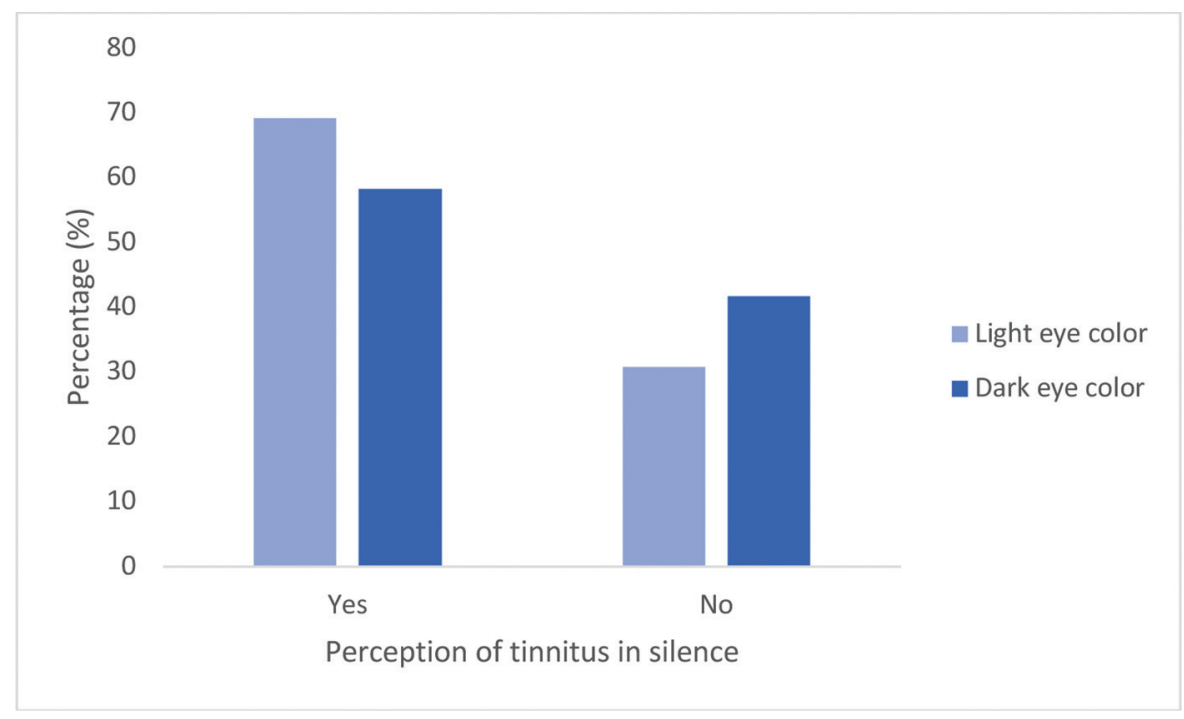

Fig. 3 Eye color and perception of tinnitus. 
than the proportion reported in the study by Heller and Bergman, ${ }^{18} 1953$ (94\%). This may be because the participants in the present study were younger and were confirmed to have normal hearing thresholds to exclude any confounding factors that may be introduced by hearing impairment. In the study by Heller and Bergman, ${ }^{18} 1953$, the participants were older, ranging from 18 to 60 years in age, and were not objectively confirmed to have normal hearing thresholds. The proportion of participants who perceived tinnitus in the present study is also lower than the proportion reported by Del Bo et al., ${ }^{17}$ 2008, in whose study an initial $83 \%$ of the participants reported the perception of tinnitus sounds in silence, with an increase to $92 \%$ when a non-functioning loudspeaker was present in the sound proof booth. This may be attributed to the difference in the instructions given to the participants. Whereas Del Bo et al., ${ }^{17} 2008$ instructed the participants to listen for a sound, in the present study, no attention was drawn to the auditory system, and the participants were instructed to sit quietly and report any experience after the period of silence. Therefore, the higher proportion of tinnitus perception reported in the study by Del Bo et al., ${ }^{17} 2008$, may be attributed to the use of auditory attention in their study. Tinnitus perception in silence appears to be enhanced by auditory attention. ${ }^{36}$

Eye Color, Race and Perception of Tinnitus in Silence In the present study, tinnitus perception did not show a significant variation regarding the different races or eye colors. Our findings of no racial differences in the perception of tinnitus in silence are contrary to those reported by Tucker et al., ${ }^{19} 2005$. This difference is unlikely to be attributed to our sample size being smaller than the one in that study. In the study by Tucker et al., ${ }^{19} 2005$, they had 120 participants with an effect size that was double what we observed in the present study. They reported that $78 \%$ of the Caucasians and $38 \%$ of the African American participants perceived tinnitus in silence. The relative risk of tinnitus perception in their study was 2.1 times higher in Caucasians than in African Americans, while it was 1.4 times higher in the present study. The odds of perceiving tinnitus in silence was 6 times higher in Caucasians than African Americans in the study by Tucker et al., ${ }^{19} 2005$. However, in the present study, the odds of perceiving tinnitus were just two times higher in Caucasians than in African Americans. The observed differences between the findings of the present study and those of Tucker et al., ${ }^{19} 2005$, may imply that any role race might play in tinnitus perception varies widely within the population, and requires further investigation.

To the best of our knowledge, very few researchers have studied the racial differences in the incidence and prevalence of tinnitus. Shargorodsky et al., ${ }^{8}$ 2010, reported that nonHispanic Caucasians were more likely to experience persistent tinnitus, while Cooper, ${ }^{37} 1994$, reported that a greater proportion of African Americans had bothersome tinnitus; however, in the study by Cooper, ${ }^{37} 1994$, the researcher did not ask about non-bothersome tinnitus. Reports from studies on racial differences in hearing impairment imply that the odds of having hearing loss is lower in African Americans than in Caucasians, and in dark-skinned Hispanics in than light-skinned Hispanics. ${ }^{38-40}$ Some researchers have also reported that age-related hearing loss is more common in Caucasians than in African Americans. ${ }^{39,41}$ Although these studies imply that melanin may play a protective role against hearing loss and age-related changes in the auditory pathway, they did not look at the differences in terms of tinnitus perception. It is possible that the protective effects of melanin are activated in pathological changes, such as hearing loss, but not physiological responses to silence, as examined in the present study. This may explain why studies have shown that the protective effect of melanin in noise-induced hearing loss is only observed at damaging levels of loud noise. ${ }^{34,42}$ In future studies, the level of cutaneous melanin in the participants could be determined using spectrophotometry technology. Additionally, a future investigation could examine the relationship between variations of pigmentation-related genes and tinnitus perception.

The risk of perceiving temporary tinnitus in silence was 1.2 times higher in light-eyed group than in those with the dark eyes. However, there was no significant difference between the proportion of light- and dark-eyed subjects who perceived tinnitus in silence. This may be a consequence of the grouping method used, in which the participants with intermediate levels of melanin concentration in the iris were grouped along with the blue-eyed subjects who had the least amount of melanin. Da Costa et al., ${ }^{34}$ 2008, and Sallustio et al., ${ }^{43} 1998$, used the same method in the grouping of their subjects. Both observed that dark-eyed subjects were less likely to develop noise-induced hearing loss than light-eyed subjects, but this difference was only significant in the study by Da Costa et al., 2008. ${ }^{34}$ Hannula et al., 2012, ${ }^{44}$ observed the opposite, that dark-eyed respondents with a history of noise exposure were more likely to have hearing impairment than light-eyed respondents. However, they did not control for the level of noise or duration of noise exposure. Another explanation could be that the protective effects of melanin may only be evident when the ear has undergone a challenge or injury. ${ }^{42}$ Its effect seems to be more evident at higher noise levels; therefore, authors report that pure-tone thresholds do not differ in normal-hearing individuals with different iris pigmentations who have not been exposed to an acoustic challenge that would cause auditory stress. ${ }^{34,45,46}$ The same might be true for the role of melanin in the emergence of tinnitus in silence. It is likely that the temporary absence of acoustic stimulation during the period of silence resulted in a compensatory increase in the burst firing rate, burst duration and spikes per burst in the central auditory pathway. ${ }^{12}$ This increase in gain is likely to be responsible for the perception of tinnitus by some of the participants, and melanin may not have a role in this pathway. ${ }^{47-49}$ However, we did not measure the activity in the central auditory pathway, and are only able to hazard a guess as to the mechanisms behind the perception of tinnitus among the study participants.

One limitation of the present study is the reliance on reports from participants for tinnitus characteristics. Examining individuals with normal hearing thresholds gives us an 
idea of the relationship between melanin and tinnitus emergence in apparently normal auditory systems, it also offers a glimpse into the response of the auditory system to reduced auditory stimulation, but it may not be generalizable to all patients with tinnitus.

The results from the present study offer a foundation for further exploration of the role of melanin in the epidemiology of tinnitus. Our results indicate that more research is needed to understand the role of melanin as a risk factor in tinnitus emergence. Research into the risk factors involved in tinnitus perception will help clinicians counsel tinnitus patients and provide explanation for some of the previously unexplained cases of tinnitus perception in individuals with apparently normal auditory systems.

\section{Conclusion}

The present study shows that 3 out of 5 individuals with normal hearing thresholds and without a history of tinnitus are likely to perceive tinnitus when they are in sustained silence. However, this perception did not differ by eye color or race. A greater knowledge of the factors that influence tinnitus emergence and perception will be useful in the counselling of tinnitus patients and in understanding the factors that determine tinnitus perception or absence in silence. The results from the present study imply that although melanin has been shown to play a role in the protection of the ear against noise trauma and the effects of age-related hearing loss, its role in the emergence of tinnitus needs further investigation. To further explore the role of melanin in tinnitus emergence following reduced auditory stimulation, it may be beneficial to look at the response of those with the smallest amount of inner-ear melanin and those with the greatest amount. A comparison of the response of individuals with blue eyes and those with brown eyes may provide a clearer glimpse into the role of melanin in the emergence of tinnitus in silence. Studies on tinnitus in silence have shown that a greater proportion of participants perceived tinnitus when they were instructed to direct their attention to the auditory system. ${ }^{17,36}$ Therefore, future studies focused on attention and awareness as possible factors contributing to the emergence of tinnitus in reduced auditory stimulation may help expand our knowledge of the risk factors for tinnitus perception. These can be obtained by comparing late auditory-evoked responses in those that perceive tinnitus and those who do not perceive tinnitus in silence. Counselling is an integral component of tinnitus therapy, and a more comprehensive idea of the risk factors behind tinnitus perception will aid clinicians in providing answers to tinnitus patients.

\section{Conflict of Interests}

The authors have no conflict of interests to delare.

\section{References}

1 Langguth B, Kreuzer PM, Kleinjung T, De Ridder D. Tinnitus: causes and clinical management. Lancet Neurol 2013;12(09): 920-930

2 Savage J, Waddell A. Tinnitus. BMJ Clin Evid. 2014:2014
3 Baguley D, McFerran D, Hall D. Tinnitus. Lancet 2013;382 (9904):1600-1607

4 Langguth $B$. A review of tinnitus symptoms beyond 'ringing in the ears': a call to action. Curr Med Res Opin 2011;27(08):1635-1643

5 Schlee W, Kleinjung T, Hiller W, Goebel G, Kolassa IT, Langguth B. Does tinnitus distress depend on age of onset? PLoS One 2011;6 (11):e27379

6 Hoffman H, Reed G. Tinnitus: Theory and Management. In: Snow JB, editor. Epidemiology of tinnitus. Lewiston, NY: B C Decker; 2004

7 Bhatt JM, Lin HW, Bhattacharyya N. Prevalence, Severity, Exposures, and Treatment Patterns of Tinnitus in the United States. JAMA Otolaryngol Head Neck Surg 2016;142(10):959-965

8 Shargorodsky J, Curhan GC, Farwell WR. Prevalence and characteristics of tinnitus among US adults. Am J Med 2010;123(08): 711-718

9 Newman CW, Sandridge SA, Bea SM, et al. Tinnitus: patients do not have to 'just live with it'. Cleve Clin J Med 2011;78(05): 312-319

10 Granjeiro RC, Kehrle HM, de Oliveira TS, Sampaio AL, de Oliveira CA. Is the degree of discomfort caused by tinnitus in normalhearing individuals correlated with psychiatric disorders? Otolaryngol Head Neck Surg 2013;148(04):658-663

11 Axelsson A, Ringdahl A. Tinnitus-a study of its prevalence and characteristics. Br J Audiol 1989;23(01):53-62

12 Noreña AJ, Eggermont JJ. Changes in spontaneous neural activity immediately after an acoustic trauma: implications for neural correlates of tinnitus. Hear Res 2003;183(1-2):137-153

13 Zhang Z, Marro SG, Zhang Y, et al. The fragile X mutation impairs homeostatic plasticity in human neurons by blocking synaptic retinoic acid signaling. Sci Transl Med 2018;10(452):eaar4338

14 Lyttkens L, Larsson B, Göller H, Englesson S, Stahle J. Melanin capacity to accumulate drugs in the internal ear. A study on lidocaine, bupivacaine and chlorpromazine. Acta Otolaryngol 1979;88(1-2):61-73

15 Lyttkens L, Larsson B, Wästerström SA. Local anaesthetics and tinnitus. Proposed peripheral mechanism of action of lidocaine. ORL J Otorhinolaryngol Relat Spec 1984;46(01):17-23

16 Levine RA, Oron Y. Tinnitus. Handb Clin Neurol 2015;129:409-431

17 Del Bo L, Forti S, Ambrosetti U, et al. Tinnitus aurium in persons with normal hearing: 55 years later. Otolaryngol Head Neck Surg 2008;139(03):391-394

18 Heller MF, Bergman M. Tinnitus aurium in normally hearing persons. Ann Otol Rhinol Laryngol 1953;62(01):73-83

19 Tucker DA, Phillips SL, Ruth RA, Clayton WA, Royster E, Todd AD. The effect of silence on tinnitus perception. Otolaryngol Head Neck Surg 2005;132(01):20-24

20 Sun DQ Zhou X, Lin FR, Francis HW, Carey JP, Chien WW. Racial difference in cochlear pigmentation is associated with hearing loss risk. Otol Neurotol 2014;35(09):1509-1514

21 Wolff D. Melanin in the Inner Ear. Arch Otolaryngol 1931;14(02): 195-211

22 Navarrete CM, Ruah CB, Schachern P, Paparella MM. Normal and metastatic melanin in the temporal bone. Am J Otolaryngol 1995; 16(01):33-39

23 White D, Rabago-Smith M. Genotype-phenotype associations and human eye color. J Hum Genet 2011;56(01):5-7

24 Bonaccorsi P. [The color of the iris as a "test" in the quantitative estimation, in man, of the melanin concentration in the stria vascularis]. Ann Laringol Otol Rinol Faringol 1965;64(06):725-738

25 Dubey S, Roulin A. Evolutionary and biomedical consequences of internal melanins. Pigment Cell Melanoma Res 2014;27(03): 327-338

26 Breathnach AS. Extra-cutaneous melanin. Pigment Cell Res 1988; 1(04):234-237

27 Nicolaus BJ. A critical review of the function of neuromelanin and an attempt to provide a unified theory. Med Hypotheses 2005;65 (04):791-796 
28 Ohlemiller KK, Rice ME, Lett JM, Gagnon PM. Absence of strial melanin coincides with age-associated marginal cell loss and endocochlear potential decline. Hear Res 2009;249(1-2):1-14

29 Xiong $\mathrm{M}$, He Q Lai $\mathrm{H}$, Wang J. Oxidative stress in spiral ganglion cells of pigmented and albino guinea pigs exposed to impulse noise. Acta Otolaryngol 2011;131(09):914-920

30 Murillo-Cuesta S, Contreras J, Zurita E, et al. Melanin precursors prevent premature age-related and noise-induced hearing loss in albino mice. Pigment Cell Melanoma Res 2010;23(01):72-83

31 Gill SS, Salt AN. Quantitative differences in endolymphatic calcium and endocochlear potential between pigmented and albino guinea pigs. Hear Res 1997;113(1-2):191-197

32 Meyer zum Gottesberge-Orsulakova A. Melanin in the inner ear: micromorphological and microanalytical investigations. Acta Histochem Suppl 1986;32:245-253

33 Barrenäs ML, Lindgren F. The influence of eye colour on susceptibility to TTS in humans. Br J Audiol 1991;25(05):303-307

34 Da Costa EA, Castro JC, Macedo ME. Iris pigmentation and susceptibility to noise-induced hearing loss. Int J Audiol 2008;47(03): 115-118

35 Esmaili AA, Renton J. A review of tinnitus. Aust J Gen Pract 2018; 47(04):205-208

36 Knobel KA, Sanchez TG. Influence of silence and attention on tinnitus perception. Otolaryngol Head Neck Surg 2008;138(01): $18-22$

37 Cooper JC Jr. Health and Nutrition Examination Survey of 197175: Part II. Tinnitus, subjective hearing loss, and well-being. J Am Acad Audiol 1994;5(01):37-43

38 Lin FR, Maas P, Chien W, Carey JP, Ferrucci L, Thorpe R. Association of skin color, race/ethnicity, and hearing loss among adults in the USA. J Assoc Res Otolaryngol 2012;13(01):109-117
39 Lin FR, Thorpe R, Gordon-Salant S, Ferrucci L. Hearing loss prevalence and risk factors among older adults in the United States. J Gerontol A Biol Sci Med Sci 2011;66(05):582-590

40 Agrawal Y, Platz EA, Niparko JK. Prevalence of hearing loss and differences by demographic characteristics among US adults: data from the National Health and Nutrition Examination Survey, 1999-2004. Arch Intern Med 2008;168(14):1522-1530

41 Helzner EP, Cauley JA, Pratt SR, et al. Race and sex differences in age-related hearing loss: the Health, Aging and Body Composition Study. J Am Geriatr Soc 2005;53(12):2119-2127

42 Kleinstein RN, Seitz MR, Barton TE, Smith CR. Iris color and hearing loss. Am J Optom Physiol Opt 1984;61(03):145-149

43 Sallustio V, Portalatini P, Soleo L, et al. Auditory dysfunction in occupational noise exposed workers. Scand Audiol Suppl 1998; 48:95-110

44 Hannula S, Bloigu R, Majamaa K, Sorri M, Mäki-Torkko E. Ear diseases and other risk factors for hearing impairment among adults: an epidemiological study. Int J Audiol 2012;51(11):833-840

45 Cunningham DR, Norris ML. Eye color and noise-induced hearing loss: a population study. Ear Hear 1982;3(04):211-214

46 Roche AF, Mukherjee D, Chumlea WC, Siervogel RM. Iris pigmentation and AC thresholds. J Speech Hear Res 1983;26(01):151-154

47 Eggermont JJ, Tass PA. Maladaptive neural synchrony in tinnitus: origin and restoration. Front Neurol 2015;6:29

48 Henry JA, Roberts LE, Caspary DM, Theodoroff SM, Salvi RJ. Underlying mechanisms of tinnitus: review and clinical implications. J Am Acad Audiol 2014;25(01):5-22, quiz 126

49 Munguia R, Pienkowski M, Eggermont JJ. Spontaneous firing rate changes in cat primary auditory cortex following long-term exposure to non-traumatic noise: tinnitus without hearing loss? Neurosci Lett 2013;546:46-50 